\title{
Multiscale Models of Quantum Dot Based Nanomaterials and Nanodevices for Solar Cells
}

\author{
Alexander I. Fedoseyev ${ }^{1}$, Marek Turowski ${ }^{1}$, Ashok Raman ${ }^{1}$, Qinghui Shao ${ }^{2}$, \\ and Alexander A. Balandin ${ }^{2}$ \\ ${ }^{1}$ CFD Research Corporation (CFDRC), 215 Wynn Drive, Huntsville, AL 35805 \\ \{aif, mt, ar2\}@efdrc.com \\ ${ }^{2}$ Nano-Device Laboratory, Department of Electrical Engineering, University of California - \\ Riverside, Riverside, California 92521 \\ \{qshao, alexb\}@ee.ucr.edu
}

\begin{abstract}
NASA future exploration missions and space electronic equipment require improvements in solar cell efficiency and radiation hardness. Novel nano-engineered materials and quantum-dot (QD) based photovoltaic devices promise to deliver more efficient, lightweight, radiation hardened solar cells and arrays, which will be of high value for the long term space missions. We describe the multiscale approach to the development of Technology Computer Aided Design (TCAD) simulation software tools for QD-based semiconductor devices, which is based on the drift - diffusion and hydrodynamic models, combined with the quantum-mechanical models for the QD solar cells.
\end{abstract}

Keywords: Nanostructured solar cell, quantum dot, photovoltaic, nanostructures, hydrodynamics, drift-diffusion, multiscale, computer-aided design, intermediate band solar cells.

\section{Introduction}

The novel modeling and simulation tools which, include multiscale, fluid and quantum models for the quantum-dot-based nanostructures, help one to better understand and predict behavior of nano-devices and novel materials in space environment, assess technologies, devices, and materials for new electronic systems [1]. The QD models are being integrated into our photonic-electronic device simulator NanoTCAD $[2,3]$, which can be useful for the optimization of QD superlattices as well as for the development and exploring of new solar cell designs.

A prototype structure for the modeling of the quantum-dot superlattice (QDS)-based photovoltaic (PV) cell is shown in Fig. 1. The basic element of this PV cell is a stack of quantum dots arrays, referred to as QDS. The QDS can be implemented on $\mathrm{Si} / \mathrm{Ge}$ or other material systems including III-Vs group materials such as GaAs. The QDS forms an intrinsic layer in a regular $n-i-p(p-i-n)$ solar cell configuration. Quantum confinement of charge carriers (electrons and holes) in variable-size quantum dots, which form the i-layer, increases the effective band gap of the material. The quantum dot size variation allows one to optimize absorption at different wavelengths and create a multicolor quantum PV cell with estimated efficiency greater than 50\% [4]. 


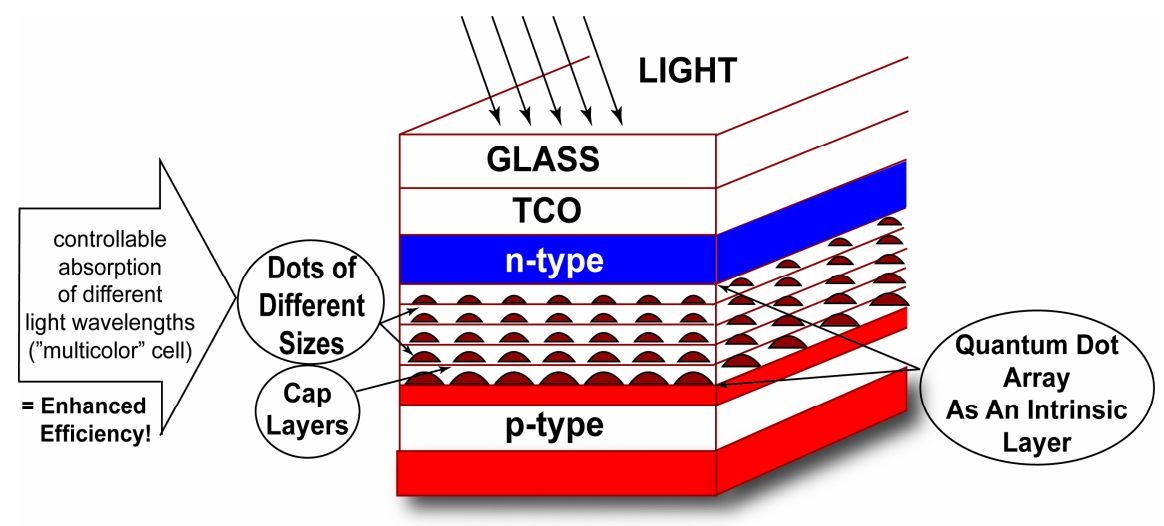

Fig. 1. Schematic structure of the PV cell based on the quantum dot superlattice (QDS), which is used as a prototype for the development of the PV cell simulation tools. The structure contains a stack of multiple quantum-dot arrays with the variable dot size, which maximizes absorption of the different light wavelengths in a controllable way.

\section{Multiscale Approach for Efficient Solution of Quantum and Fluid Level Models}

Drift-diffusion (DD) based models have a long and fruitful history in applications to 3D simulations of not only modern electronic devices, but also to optoelectronic ones. In recent years, however, a new class of devices has been emerging and they require tools that include quantum effects (quantum well, tunnel junction, Schottky contact, quantum dot nanostructures etc.) and also allow for efficient numerical implementation. We have proposed and tested in 3D device simulator NanoTCAD a number of the reduced models for quantum scale of the problem, which have been successfully verified on experimental and numerical data [3]. For example, for the quantum well

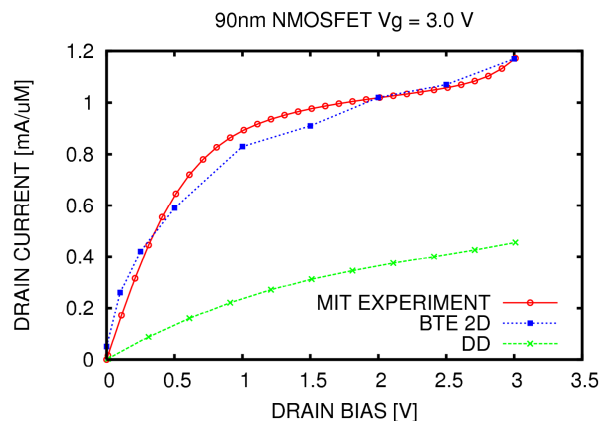

(a)

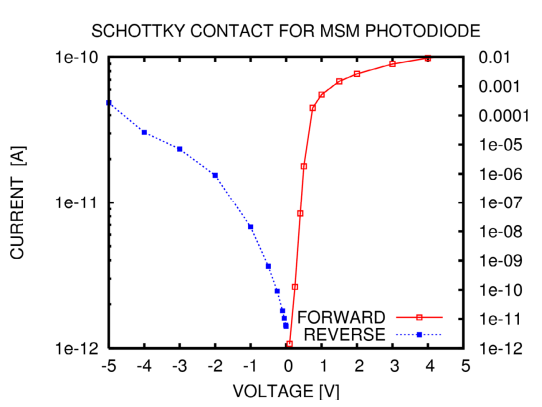

(b)

Fig. 2. (a) Comparison of I-V characteristics of MIT NMOSFET transistor calculated with BTE and DD versus experimental data [4]. (b) CFDRC modeling for Schottky-based Philips MSM photodetector [6], I-V characteristic for reverse and forward bias. A good agreement has been obtained for the forward bias and correct trend for the reverse bias. 
within 3D electronic device, we have proposed "tunneling mobility", which was calculated from pure quantum problem for carriers tunneling through the barrier. That approach has shown good accuracy and efficiency, compared to Wigner function method and Boltzmann transport equation with quantum corrections [3].

In the current problem we are developing the approach similar to one successfully developed for the incorporation of kinetic effects into the 3D drift-diffusion (DD) model. The problem for the device region with strong kinetic effects has been solved using the 4D Boltzmann transport equation (BTE), 3D geometry, and 1D energy space, and macroscopic transport coefficient calculated from the kinetic probability distribution function (see Fig.2). The details of the solution are reported elsewhere [5].

The modeling of the photovoltaic cell with QDS (see Fig. 3 (a)) is conducted with 3D NanoTCAD device simulator, which uses the quantum level computed transport parameters for the i-layer, the device region containing quantum dot superlattice, while for other device regions, the classical DD models will be used. Typical I-V curves for photovoltaic device, silicon p-i-n solar cell, calculated with NanoTCAD are shown in Fig. 3(b). f
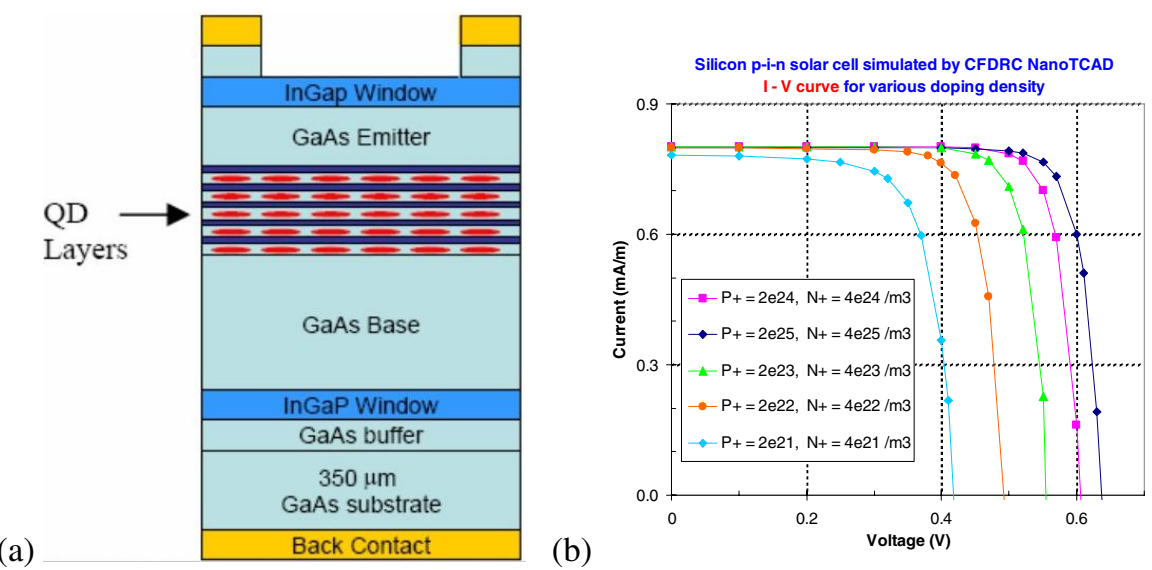

Fig. 3. (a) Typical photovoltaic cell with quantum dot superlattice and (b) our results for solar cell simulation with NanoTCAD [2]

\section{Models Implemented with 3D Device Simulator Nanotcad}

The multiscale photovoltaic (PV) models discussed above are being integrated within the advanced software tool NanoTCAD, which is a 3D device simulator developed and commercialized by CFD Research Corporation [2]. This integration provides a user-friendly interface and a large database of the semiconductor material properties available in NanoTCAD. It also makes possible a complete PV-cell simulation including both quantum and classical models for the appropriate PV-cell elements, both DC and transient regimes, etc. The models are currently being extended to incorporate simulation of the electron-phonon transport in QDS made of semiconductors with 
both cubic and hexagonal crystal lattice, e.g., InAs/GaAs, Ge/Si, CdSe, ZnO. The drift-diffusion model implemented in NanoTCAD is described below.

\subsection{Drift-Diffusion Model}

Drift-diffusion models are formulated based on continuity equations for electrons and holes and Poisson equation for electrostatic potential. They are able to provide good comparison with experimental data for transistors with channel length down to $15 \mathrm{~nm}$.

Conservation of charge for electrons is represented by continuity equation

$$
q \frac{\partial n}{\partial t}+\nabla \cdot J=q R
$$

and similarly for holes as

$$
q \frac{\partial p}{\partial t}+\nabla \cdot J p=-q R
$$

where the electron current is

$$
J_{n}=-q \mu_{n}\left(U_{T} \nabla n-n \nabla \Psi\right),
$$

and hole current is

$$
J_{p}=-q \mu_{p}\left(U_{T} \nabla p-p \nabla \Psi\right)
$$

Here $n$ and $p$ are electron and hole densities $\left[1 / \mathrm{cm}^{3}\right], \Psi$ is electrostatic potential [V], $q$ is carrier charge (electron charge $e$ ), $U_{T}=\frac{k_{B} T}{q}$, and diffusion coefficients are $D_{n}=U_{T} \mu_{n}$ and $D_{p}=U_{T} \mu_{p}\left[\mathrm{~cm}^{2} / \mathrm{s}\right]$.

Electron and hole mobilities $\mu_{n}, \mu_{n}$ are calculated parameters (models depend on the material, device, or calculated from quantum or kinetic level problems).

Electrostatic potential which appears in current equations is governed by Poisson equation

$$
\nabla \varepsilon \nabla \Psi=q(n-p-C),
$$

where $\Psi$ is electrostatic potential [V], $\varepsilon$ is dielectric constant, and $C$ is a doping, $C=N_{D^{-}}^{+} N_{A}^{-}$.

\subsection{Boundary Conditions}

Boundary conditions for $n, p$, and $\Psi$ are shown below for the example of Ohmic contact. At the Ohmic contact we assume thermal equilibrium and vanishing space charge which results in 


$$
\begin{aligned}
& n \cdot p-n_{i}^{2}=0, \\
& n-p-C=0 .
\end{aligned}
$$

Solving a quadratic equation for $n, p$ we get Dirichlet conditions for $n$ and $p$ on the boundary (Ohmic contact)

$$
\begin{aligned}
& n_{0}=\frac{1}{2}\left(\sqrt{C^{2}+4 n_{i}^{2}}+C\right), \\
& p_{0}=\frac{1}{2}\left(\sqrt{C^{2}+4 n_{i}^{2}}-C\right) .
\end{aligned}
$$

The boundary potential at an Ohmic contact is the sum of the externally applied potential (voltage) $V_{C}(t)$ and the so called built-in potential, which is produced by doping

$$
\Psi=\Psi_{b i}+V_{C}^{(t)}
$$

The built-in potential is

$$
\Psi_{b i}=U_{T} \ln \left[\frac{C(x)+\sqrt{C^{2}+4 n_{i}^{2}}}{2 n_{i}}\right],
$$

where the intrinsic concentration $n_{i}$ is:

$$
n_{i}=\sqrt{n \cdot p}
$$

\subsection{Solution of Governing Equations for Drift-Diffusion Based Model}

Governing equations (1) to (4), (5) are discretized by finite volume method and solved simultaneously using the Newton technique, to ensure a good convergence. In NanoTCAD, we use a high-performance iterative linear solver CNSPACK, developed by Fedoseyev [7] . CNSPACK uses a high order preconditioning by incomplete decomposition to ensure the accuracy, stability and convergence of the simulations.

The linear algebraic system is solved in CNSPACK using a CGS-type iterative method with preconditioning by the incomplete decomposition of the matrix. Comparing the CGS and GMRES methods [8] , [9] in different tests, it was found that both methods converge well, if a good preconditioner is used. The CGS method needs less memory to store only eight work vectors.

To reduce the memory requirements, a compact storage scheme for matrices is used in CNSPACK. It stores only the nonzero matrix entries. The incomplete decomposition $(I D)$ used for preconditioning, is constructed as a product of triangular and diagonal matrices, $\boldsymbol{P}=\boldsymbol{L D} \boldsymbol{U}$. To avoid diagonal pivot degeneration, the Kershaw 
diagonal modification is used [10]. If the value of diagonal element becomes small during the construction of preconditioning matrix, i.e. $\left|a_{i i}\right|<\alpha=\sqrt{2^{-t} \sigma \mu}$, the diagonal $a_{i i}$ is replaced by $\alpha$. Here $\sigma$ and $\mu$ are the maximum magnitudes of current row $_{i}$ and column $_{i}$ elements, and $2^{-t}$ is a machine precision $(t$ bits in mantissa, see the proof of eligibility in [10]).

For the first order $I D$, the matrix $P$ has the same non-zero entry pattern as the original matrix. For a second order or higher $I D$, matrix $\mathrm{P}$ has one or more additional entries near the locations of the non-zero matrix entries, where the original matrix entries are zeros.

Fig. 4 shows a typical dependence of total memory and CPU time for NanoTCAD simulations of $3 \mathrm{D}$ devices using unstructured meshes. Compared to linear solver, used in other commercial device simulators, NanoTCAD solver uses dramatically less memory (by more than one order of magnitude), and CPU time is also smaller by one order of magnitude compared to [11] for similar problem size / number of unknown.

NanoTCAD simulator can solve a transient 3D multi-branched ion strike problem with 100,000 node unstructured mesh within 512MB memory. It can solve the problem with 3D unstructured mesh for up to 500,000 nodes within 2GB memory.

Typical steady state solution for 3D semiconductor device / solar cell takes 5 to 10 Newton iterations to reach the ten order reduction of initial residual. This corresponds to very short computational time of NanoTCAD (typically a couple of minutes or less). Large transient problems, for example, simulation of radiation effects, produces

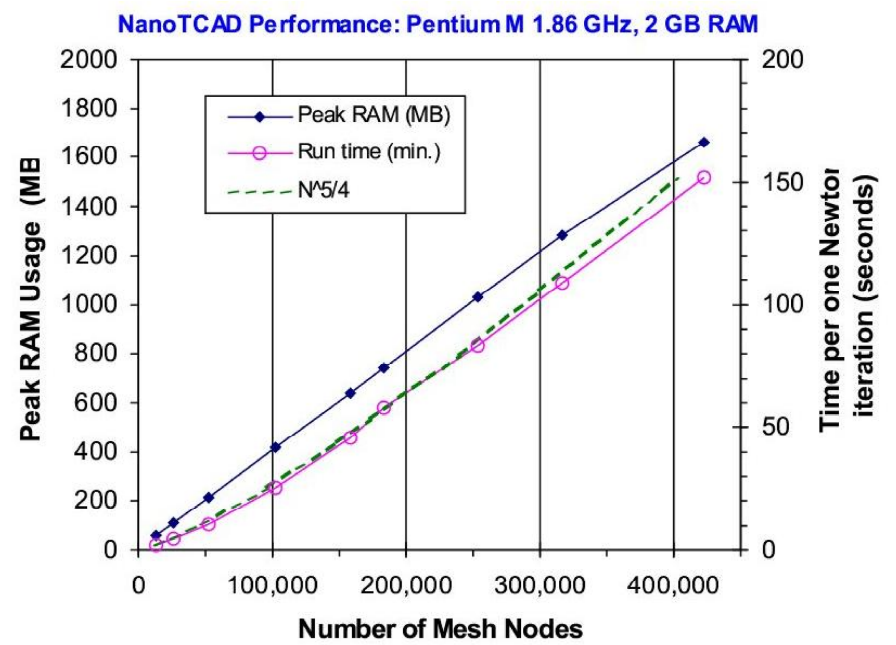

Fig. 4. NanoTCAD simulator performance for modeling 3D devices: memory, solid curve with diamonds(in MB), and CPU time, solid curve with circles (in secs per Newton iteration) versus number of mesh nodes. One can see that the dependence is almost linear for both memory and CPU time, and close to theoretical estimate for the CPU time, $T=O\left(N^{5 / 4}\right)$ (dashed curve). 
by high-energy ion strike through the electron device (not relevant to the problems, considered in this paper, please see another our paper [15]), may need more times (few days).

This efficient linear solver made possible to use adaptive unstructured 3D mesh generation for a transient 3D multi-branched ion strike problem.

\subsection{Solution for Carrier Transport in 3D Quantum Dot Superlattice}

We use the Lazarenkova-Balandin model for computation of the electron energy spectra of 3D regimented quantum dot superlattice, which has been proposed earlier [12, 13].

Schematic structure of the quantum dot superlattice is shown in Fig. 5. The electron spectrum is analyzed by using one-electron Schroedinger equation:

$$
\left[-\frac{\hbar^{2}}{2} \nabla_{\mathbf{r}} \frac{1}{m^{*}} \nabla_{\mathbf{r}}+V(\mathbf{r})\right] \varphi(\mathbf{r})=E \varphi(\mathbf{r}) .
$$

Here $\varphi(\mathbf{r})$ is the electron wave function, $E$ is the electron energy, and the confining potential profile $V(\mathbf{r})$ corresponds to an infinite sequence of quantum dots of sizes $L_{x}, L_{y}, L_{z}$ separated by the barriers of thicknesses $H_{x}, H_{y}, H_{z}$. The potential $V(\mathbf{r})$ is set to zero in the barrier region while inside the quantum dot it is equal to the band offset in the conduction (or valence) band of the considered material system taken with a negative sign.

The solution of the Schroedinger equations can have semi-analytical approximation using the simplified potential model [13]. Fig. 5(b) shows the results of quantum level approximation only, for the efficiency of the PV cell depending of the QDS dimensions, for a particular material.

\subsection{Coupling of Quantum and Fluid Level Models of Electronic Device}

Electron and hole mobilities $\mu_{n}, \mu_{n}$ in Eq. (3) and (4) are calculated parameters . Mobility depends on the material, device structure for the fluid transport dominated device region, or calculated from quantum or kinetic level problems for the device regions where the fluid model is not valid. Then, this calculated "quantum" or "kinetic" mobility is used in Eq. (3), (4) for the fluid model. Finally, the whole device is simulated with fluid level model, using the transport coefficient from quantum level, where this is needed only (in the device region with dominated quantum transport, like quantum dot layers in Fig. 3).

For example, for the quantum dot layers in Fig. 3, we calculate the "quantum" mobility in the device region with quantum dominated transport as follows:

$$
\mu_{q}=\sum \mu_{i} \frac{n_{s i}}{n_{s}}
$$

where $n_{s i}=\frac{m}{\pi \hbar^{2}} \int_{E_{s i}}^{\infty} f(E) d E$ is the concentration of electrons in subband $i$ with the bottom energy $E_{s i}, f(E)$ is Fermi-Dirac distribution function, subband mobility 
$\mu_{i}=\frac{e}{m}\left\langle\frac{1}{\tau_{E}}\right\rangle^{-1}$, where the brackets mean the average value. The electron momentum relaxation time (life time) $\tau_{i}$ in the state $E$ of subband $i$, is calculated using the subband energy levels from the solution of Eq. (13) and the scattering rates of an electron, see for example [14], p.205.

Similar approach has been successfully developed for the incorporation of kinetic effects into the 3D carrier fluid transport model. In the device region with strong kinetic effects, we solved the 4D Boltzmann transport equation (BTE), (3D geometry, plus 1D energy space), and macroscopic transport coefficient have been calculated from the kinetic probability distribution function. Results have compared well with the ones published in literature, obtained using accurate sophisticated models, like Wingner equations, and experimental data (see details in [5]).
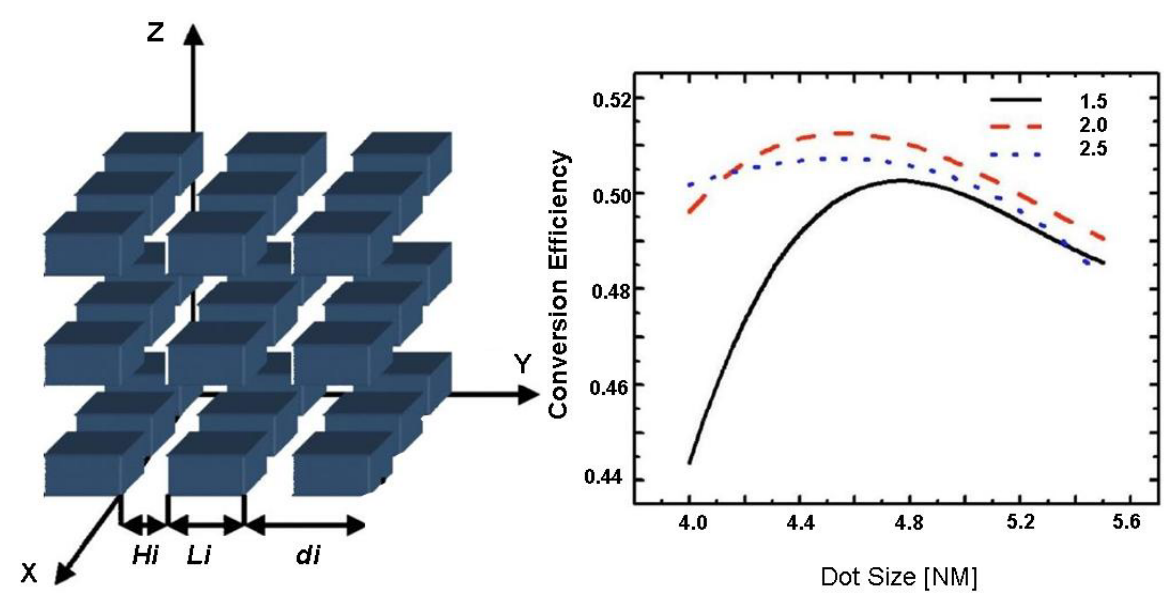

Fig. 5. (a) Schematic structure of the quantum dot superlattice in PV cell, $L_{i}, H i, d_{i}$ are dot size, inter-dot separation and QD lattice period in $i$-direction, $i=x, y, z$. (b). Photovoltaic power conversion efficiency as a function of the quantum dot size in $I n A s_{0.9} N_{0.1} / G a A s_{0.98} S b_{0.02}$ quantum dot superlattice. The results are shown for several interdot separations.

\section{Conclusions}

We have discussed how the new multiscale models for the charge carrier transport in the quantum dot superlattices are being implemented into the advanced photonicelectronic 3D device simulator NanoTCAD. Based on our successful multiscale coupling for kinetic and hydrodynamics transport levels in sub-micron semiconductor devices, we are proposing the approach of multiscale integration for quantum dot based solar cells. This include the quantum physics based model for quantum dot superlattice and classical hydrodynamics / drift diffusion model for carrier transport in 3D semiconductor devices. 


\section{References}

1. Balandin, A.A., Lazarenkova, O.L.: Mechanism for thermoelectric Fig.-of-merit enhancement in regimented quantum dot superlattices. App. Phys. Let. 82, 415 (2003)

2. CFDRC, NanoTCAD website (2006), http://www.cfdrc.com/bizareas/microelec/micro_nano/

3. Fedoseyev, A.I., Turowski, M., Wartak, M.S.: Kinetic and Quantum Models for Nanoelectronic and Optoelectronic Device Simulation. J. Nanoelectr. Optoelectr. 2, 234-256 (2007)

4. Shao, Q., Balandin, A.A., Fedoseyev, A.I., Turowski, M.: Intermediate-band solar cells based on quantum dot supracrystals. App. Phys. Let. 91(16) (2007)

5. Fedoseyev, A.I., Kolobov, V., Arslanbekov, R., Przekwas, A.: Kinetic simulation tools for nano-scale semiconductor devices. Microelectronic Eng. 69, 577-586 (2003)

6. Seto, M., Leduc, J.-V., Lammers, A.M.F.: Al-n-Si Double Schottky Photodiodes for Optical Storage Systems. In: Proc. ESSDERC 1997 (1997)

7. Fedoseyev, A.I., Bessonov, O.A.: Iterative solution for large linear systems for unstructured meshes with preconditioning by high order incomplete decomposition. Comput. Fluid Dynam. J. 10, 296 (2001)

8. Wesseling, P., Sonneveld, P.: Numerical experiments with a multiple grid and a preconditionned Lanczos type methods. Lecture Notes in Mathematics, vol. 771, pp. 543-562. Springer, Berlin (1980)

9. Saad, Y.: GMRES: A generalized minimal residual algorithm for solving nonsymmetric linear systems. SIAM J. Sci. Statist. Comput. 7, 856 (1986)

10. Kershaw, D.S.: The incomplete Cholesky-conjugate gradient method for the iterative solution of systems of linear equations. J. Comput. Phys. 2, 43 (1978)

11. Schenk, O., Rllin, S., Hagemann, M.: Recent advances in sparse linear solver technology for semiconductor device simulation matrices. In: Proc. Int. Conf. Simulation of Semi. Processes and Devices, Boston, MA, USA, vol. 103 (2003)

12. Lazarenkova, O.L., Balandin, A.A.: Electron and photon energy spectra in a threedimensional regimented quantum dot superlattice. Phys. Rev. 66, 245-319 (2002)

13. Balandin, A.A., Lazarenkova, O.L.: Miniband formation in a quantum dot crystal. J. App. Phys. 89, 5505 (2001)

14. Landau, L.D., Lifshitz, E.M., Pitaevskii, L.P.: Statistical Physics, Science, p. 387 (1980)

15. Fedoseyev, A.I., Turowski, M., Raman, A., Alles, M.L., Weller, R.A.: Multiscale Numerical Models for Simulation of Radiation Events in Semiconductor Devices. In: Bubak, et al. (eds.) ICCS 2008 part II. LNCS, vol. 5102, pp. 281-290. Springer, Heidelberg (2008) 